\title{
Mudanças fonológicas obtidas no tratamento de sujeitos comparando diferentes modelos de terapia****
}

\author{
Phonological changes obtained in the treatment of subjects \\ comparing different therapy models
}

\author{
Marizete Ilha Ceron* \\ Márcia Keske-Soares** \\ Giséli Pereira de Freitas*** \\ Marileda Barichello Gubiani***
}

\begin{abstract}
*Fonoaudióloga. Mestre em Distúrbios da Comunicação Humana pela Universidade Federal de Santa Maria (UFSM). Residente do Programa de Residência Multiprofissional Integrada em Sistema Público de Saúde da UFSM. Endereço para correspondência: Rua Bentevi, 215 - Santa Maria - RS CEP 97035-130.

(marizeteceron@hotmail.com).

**Fonoaudióloga. Doutora em Linguística Aplicada pela Pontifícia Universidade Católica do Rio Grande do Sul. Professora Adjunta do Curso de Graduação em Fonoaudiologia e do Programa de Pós-Graduação em Distúrbios da Comunicação Humana da UFSM.

***Fonoaudióloga. Mestranda em Distúrbios da Comunicação Humana pela UFSM. Bolsista pela Coordenação de Aperfeiçoamento de Pessoal de Nível Superior (Capes).

****Trabalho Realizado no Curso de Fonoaudiologia da UFSM.
\end{abstract}

Artigo Original de Pesquisa

Artigo Submetido a Avaliação por Pares

Conflito de Interesse: não

Recebido em 05.11.2009.

Revisado em 09.11.2010.

Aceito para Publicação em 16.11.2010.

\begin{abstract}
Background: there are many options of phonological disorder treatments that aim at improving children's communication. Aim: to analyze the Percentage of Consonants Correct-Revised, the number of acquired phonemes in the phonological system and the types of generalizations obtained in treatment, comparing different therapy models in subjects with different severity levels of phonological disorder. Method: participants were 21 children, mean age 5:7 years. All subjects underwent speech, language and hearing evaluations and additional exams. Children were then divided into groups according to therapy model and severity level of phonological disorder. The Percentage of Consonants Correct-Revised, the number of acquired phonemes and the types of generalizations were analyzed and compared within and between each therapeutic model, considering pre and post therapy evaluations. Results: it was possible to observe improvement in the three analyzed therapy models. When comparing the therapy models, the greater improvement in percentage terms was observed for the ABAB-Withdrawal and Multiple Probes Model and the Maximal Oppositions Model, even though the statistical analysis indicated no significant difference. Conclusion: the three therapy models were effective for the treatment of children with phonological disorder because they all of them provided an increase in the Percentage of Consonants Correct-Revised, in the number of acquired phonemes and in the types of analyzed generalizations.
\end{abstract}

Key Words: Articulation Disorders; Speech Therapy; Child.

\section{Resumo}

Tema: há muitas opções de tratamento para o desvio fonológico os quais buscam melhorar a comunicação das crianças. Objetivo: este estudo visa analisar o Percentual de Consoantes Corretas-Revisado, o número de fonemas adquiridos no sistema fonológico e os tipos de generalizações obtidas no tratamento, comparando diferentes modelos de terapia em sujeitos com diferentes gravidades do desvio fonológico. Método: a amostra constou de 21 crianças, com idade média de 5:7 anos. Foram realizadas as avaliações fonoaudiológicas e exames complementares. Após a realização destas avaliações, as crianças foram classificadas em grupos de acordo com o modelo de terapia e gravidade do desvio fonológico. O Percentual de Consoantes Corretas-Revisado, o número de fonemas adquiridos e os tipos de generalizações foram analisados e comparados em cada modelo e entre os modelos terapêuticos, por meio das avaliações inicial e final. Resultados: ao comparar os itens em cada modelo observaram-se evoluções nos três modelos pesquisados. Na comparação entre modelos, os maiores aumentos de percentuais encontram-se nos Modelos ABAB-Retirada e Provas Múltiplas e Oposições Máximas, apesar de a análise estatística mostrar que não há diferença significativa entre eles. Conclusão: os três modelos aplicados foram eficazes no tratamento destas crianças com desvio fonológico, pois proporcionaram um aumento no Percentual de Consoantes Corretas-Revisado, no número de fonemas adquiridos e nos tipos de generalizações pesquisados. Palavras-Chave: Transtornos da Articulação; Fonoterapia; Criança.

Referenciar este material como: 


\section{Introdução}

Uma comunicação eficiente ocorre normalmente pela linguagem oral. Algumas crianças, ainda que na faixa etária esperada e sem comprometimentos orgânicos, podem apresentar dificuldades na aquisição dos sons de sua língua. Essa dificuldade é denominada de desvio fonológico que é caracterizado por omissões e substituições de sons na fala ${ }^{1}$.

Atualmente, têm-se muitas opções de modelos terapêuticos com base fonológica para tratar essas crianças com desvios fonológicos. Alguns dos modelos descritos na literatura são: Modelo ABAB - Retirada e Provas Múltiplas², Modelo de Oposições Máximas Modificado ${ }^{3}$ e Modelo de Ciclos Modificado ${ }^{4}$.

Esses modelos buscam tornar a comunicação mais efetiva, pela reestruturação do sistema fonológico, visando à generalização $0^{3,5-10}$. A generalização é o aumento da produção correta dos fones-alvo trabalhados para outros contextos ou ambientes não-treinados ${ }^{6,11}$. Sendo também uma maneira de verificar a efetividade de cada modelo terapêutico.

Porém, ainda são poucos os trabalhos que comparam a eficácia dessas abordagens de tratamento ${ }^{5,8,10,12-13}$. Assim, o objetivo deste estudo é analisar o Percentual de Consoantes CorretasRevisado (PCC-R), o número de fonemas adquiridos no sistema fonológico e os tipos de generalizações obtidas comparando diferentes modelos de terapia em sujeitos com diferentes gravidades do desvio fonológico.

\section{Método}

O grupo de indivíduos foi constituído de 21 crianças monolíngues do português brasileiro e com desvio fonológico, sendo 11 do sexo masculino e 10 do feminino. A média de idade no início do tratamento era de 5:7. Para que houvesse a participação dos indivíduos na pesquisa (e posterior publicação dos resultados), todos os pais ou responsáveis dos mesmos, receberam informações sobre a pesquisa e assinaram o Termo de Consentimento Livre e Esclarecido. Esta pesquisa encontra-se vinculada a um projeto de pesquisa aprovado pelo Comitê de Ética em Pesquisa de uma instituição de ensino superior, sob $n^{\circ} 046 / 02$.

Os critérios de inclusão do grupo de sujeitos na pesquisa foram: diagnóstico de desvio fonológico; ausência de alterações significativas nas avaliações realizadas, exceto na fonológica; apresentar diferentes gravidades do desvio fonológico.

Para confirmar o diagnóstico de desvio fonológico e descartar outros comprometimentos que pudessem interferir no desenvolvimento da linguagem, as crianças foram submetidas às seguintes avaliações fonoaudiológicas: anamnese, linguagem compreensiva e expressiva; sistema estomatognático; discriminação auditiva, e avaliação fonológica. Além disso, todos foram submetidos a exames complementares, envolvendo avaliação otorrinolaringológica, audiológica e neurológica.

Os dados da fala de todos os sujeitos foram gravados, transcritos foneticamente e analisados pela análise contrastiva. A partir dessa análise foi possível obter o sistema fonológico de cada criança. Foram utilizados os critérios de um fonema adquirido quando ocorre em $80 \%$ ou mais das possibilidades, parcialmente adquirido quando ocorre de $40 \%$ - 79\% das vezes e não adquirido quando ocorre de $0 \%$ - 39\% das chances ${ }^{14}$.

A partir da análise contrastiva, foi calculada a gravidade do desvio fonológico através do cálculo do PCC- $\mathrm{R}^{15}$. Os percentuais de classificação utilizados foram os mesmos descritos para o PCC $^{16}$.

A partir dos resultados do PCC-R os sujeitos foram classificados em desvio grave (PCC-R $<50 \%$ ), desvio moderado-grave (51\% < PCC-R $>65 \%$ ), desvio levemoderado (66\% < PCC-R > 85\%) e desvio leve (86\% < PCC-R > 100\%). De acordo com o PCC-R, dividiu-se a amostra em dois grupos, o grupo 1 (G1) composto por nove sujeitos com desvio severo ou moderado-severo e o grupo 2 (G2) constituído por doze sujeitos com desvio médio-moderado ou médio.

Ainda os sujeitos selecionados para participar da pesquisa foram classificados em três grupos, de acordo com o modelo de terapia fonológica utilizado durante o tratamento, sendo assim distribuídos: seis submetidos ao tratamento pelo Modelo ABABRetirada e Provas Múltiplas (A); sete, ao Modelo de Oposições Modificado (O); e oito, ao Modelo de Ciclos Modificado (C).

A terapia fonológica foi realizada através de atividades lúdicas, de acordo com o interesse de cada criança, obedecendo aos procedimentos e peculiaridade de cada modelo. Foi também realizado um trabalho de orientações aos pais ou responsáveis, no final de cada sessão, no qual eram entregue cópias (em desenhos e por escrito) das palavras trabalhadas e a lista de palavras do bombardeio auditivo, recomendando que a mesma fosse lida em casa ao menos uma vez ao dia para a criança. 
No Modelo ABAB-Retirada e Provas Múltiplas foram realizados de 2 a 4 ciclos de terapia, aproximadamente de 18 a 36 sessões, conforme a necessidade da criança. No Modelo Oposições Máximas foram realizadas 20 sessões de terapia e no Modelo de Ciclos Modificado foram 2 ciclos de tratamento, aproximadamente de 12 a 24 sessões.

No final do tratamento, os sujeitos foram submetidos à reavaliação fonológica (avaliação final). Foram analisados o PCC-R, o número de sons adquiridos e os seguintes tipos de generalizações: a itens lexicais não utilizados no tratamento, para outra posição na palavra, dentro de uma classe de sons, para outras classes de sons.

A análise da evolução da terapia foi observada por meio da comparação do diferencial entre as análises contrastiva das avaliações (AI - avaliação inicial) e reavaliações (AF - avaliação final) realizadas nos diferentes modelos de terapia. Os dados foram analisados comparando os modelos terapêuticos e depois as gravidades do desvio fonológico. Estas análises foram submetidas a tratamento estatístico, utilizando o teste ANOVA considerando o nível de significância p < 0,05.

\section{Resultados}

Na Tabela 1 é apresentada a descrição da comparação do PCC-R, número de fonemas adquiridos no sistema fonológico e generalizações (a itens lexicais não utilizados, outras posições na palavra, dentro de uma classe de sons e para outras classes de sons) entre os modelos de terapia em cada grupo de gravidade do desvio fonológico.
É possível verificar grandes evoluções (diferencial entre a AI e AF) quanto aos itens analisados, apesar de não se observar diferença estatística entre os Modelos ABAB-Retirada e Provas Múltiplas, Oposições Máximas e Ciclos Modificado em relação ao PCC-R, número de fonemas adquiridos no sistema fonológico e aos tipos de generalização.

Nota-se que os maiores aumentos de percentuais encontram-se nos Modelos ABABRetirada e Provas Múltiplas e Oposições Máximas. Em ambos os grupos a análise estatística mostrou que os modelos terapêuticos não diferiram entre si quanto aos itens analisados: PCC-R, número de fonemas adquiridos e tipos generalização, sendo os três modelos efetivos no tratamento de sujeitos com diferentes gravidades do desvio fonológico.

A Tabela 2 expõe a comparação dos grupos de gravidade do desvio fonológico quanto ao PCC-R, número de fonemas adquiridos e tipos de generalização em cada modelo de terapia.

Percebe-se que houve evoluções nos três modelos pesquisados: ABAB-Retirada e Provas Múltiplas, Oposições Máximas Modificado e Ciclos Modificado.

No Modelo ABAB-Retirada e Provas Múltiplas, observou-se importantes progressos quanto aos itens analisados, porém houve diferença estatística entre os grupos, apenas no PCC-R e para a generalização dentro de uma classe de sons.

Para o Modelo Oposições Máximas Modificado apenas o PCC-R foi estatisticamente significante na comparação entre os grupos de gravidades. $\mathrm{O}$ Modelo de Ciclos Modificado foi o que menos proporcionou aquisições, não havendo diferença estatística entre os grupos (G1 e G2).

TABELA 1. Comparação das mudanças fonológicas entre os modelos fonológicos em diferentes grupos de gravidades do desvio fonológico.

\begin{tabular}{|c|c|c|c|c|c|c|c|c|}
\hline \multirow[b]{2}{*}{ Modelos } & \multicolumn{4}{|c|}{$\begin{array}{c}\text { Grupo } 1 \\
(\mathrm{~N}=9)\end{array}$} & \multicolumn{4}{|c|}{$\begin{array}{l}\text { Grupo } 2 \\
(\mathrm{~N}=12)\end{array}$} \\
\hline & $\begin{array}{c}\text { A } \\
(n=4)\end{array}$ & $\begin{array}{c}O \\
(n=3)\end{array}$ & $\begin{array}{c}C \\
(n=2)\end{array}$ & $\mathrm{P}$ & $\begin{array}{c}A \\
(n=4)\end{array}$ & $\begin{array}{c}O \\
(n=4)\end{array}$ & $\begin{array}{c}C \\
(n=4)\end{array}$ & $\mathrm{p}$ \\
\hline PCC-R & $39,5(19,8)^{\mathrm{a}}$ & $23,1(2,3)^{\mathrm{a}}$ & $6,8(1,5)^{\mathrm{a}}$ & 0,08 & $13,8(4,5)^{\mathrm{a}}$ & $9,6(6,7)^{\mathrm{a}}$ & $9,5(4,5)^{\mathrm{a}}$ & 0,46 \\
\hline número FA & $3,7(2,1)^{\mathrm{a}}$ & $3,7(2,5)^{a}$ & $0,0(0,0)^{\mathrm{a}}$ & 0,16 & $2,0(2,3)^{a}$ & $0,5(0,6)^{\mathrm{a}}$ & $0,2(0,5)^{\mathrm{a}}$ & 0,22 \\
\hline ítens lexicais não utilizados no tratamento & $60,2(25,9)^{\mathrm{a}}$ & $42,2(30,6)^{\mathrm{a}}$ & $28,0(13,3)^{\mathrm{a}}$ & 0,39 & $84,5(11,4)^{\mathrm{a}}$ & $61,9(35,3)^{\mathrm{a}}$ & $50,6(18,3)^{a}$ & 0,18 \\
\hline outras posições na palavra & $56,5(38,4)^{\mathrm{a}}$ & $53,6(34,7)^{\mathrm{a}}$ & $0,0(0,0)^{\mathrm{a}}$ & 0,20 & $32,4(38,8)^{a}$ & $51,6(39,2)^{\mathrm{a}}$ & $19,3(14,9)^{\mathrm{a}}$ & 0,42 \\
\hline dentro de uma classe de sons & $46,1(8,7)^{\mathrm{a}}$ & $48,9(39,2)^{\mathrm{a}}$ & $12,1(1,4)^{\mathrm{a}}$ & 0,25 & $20,5(18,1)^{\mathrm{a}}$ & $34,1(36,4)^{\mathrm{a}}$ & $38,3(35,0)^{\mathrm{a}}$ & 0,70 \\
\hline outras classes de sons & $44,3(29,9)^{\mathrm{a}}$ & $36,3(18,5)^{\mathrm{a}}$ & $0,0(0,0)^{\mathrm{a}}$ & 0,17 & $44,9(15,3)^{\mathrm{a}}$ & $19,2(23,0)^{\mathrm{a}}$ & $13,6(27,3)^{\mathrm{a}}$ & 0,16 \\
\hline
\end{tabular}

Legenda: PCC-R: percentual de consoantes corretas-revisado; FA: fonemas adquiridos; A: ABAB-Retirada e Provas Múltiplas; O: Oposições Máximas Modificado; C: Ciclos Modificado. 
TABELA 2. Comparação das mudanças fonológicas nos diferentes grupos de gravidades dentro de cada modelo terapêutico.

\begin{tabular}{|c|c|c|c|c|c|c|c|c|c|}
\hline & \multicolumn{3}{|c|}{$\begin{array}{l}\text { Modelo ABAB-Retirada e Provas } \\
\text { Múltiplas }(\mathrm{N}=8)\end{array}$} & \multicolumn{3}{|c|}{$\begin{array}{l}\text { Modelo de Oposições Máximas } \\
\qquad(\mathrm{N}=7)\end{array}$} & \multicolumn{3}{|c|}{$\begin{array}{l}\text { Modelo de Ciclos Modificado } \\
\qquad(\mathrm{N}=6)\end{array}$} \\
\hline & $\begin{array}{c}\mathrm{G} 1 \\
(\mathrm{~N}=4)\end{array}$ & $\begin{array}{c}\mathrm{G} 2 \\
(\mathrm{~N}=4)\end{array}$ & $\mathrm{p}$ & $\begin{array}{c}G 1 \\
(N=3)\end{array}$ & $\begin{array}{c}G 2 \\
(N=4)\end{array}$ & $\mathrm{p}$ & $\begin{array}{c}\mathrm{G} 1 \\
(\mathrm{~N}=2)\end{array}$ & $\begin{array}{c}\mathrm{G} 2 \\
(\mathrm{~N}=4)\end{array}$ & $\mathrm{p}$ \\
\hline PCCR & $39,5(19,8) a$ & $13,8(4,5) b$ & 0,04 & $23,1(2,3) a$ & $9,6(6,7) \mathrm{b}$ & 0,02 & $6,8(1,5) a$ & $9,5(4,6) \mathrm{a}$ & 0,48 \\
\hline número FA & $3,7(2,0) \mathrm{a}$ & $2,0(2,3) \mathrm{b}$ & 0,30 & $3,7(2,5) \mathrm{a}$ & $0,5(0,6) \mathrm{b}$ & 0,05 & $0,0(0,0) a$ & $0,25(0,5) \mathrm{a}$ & 0,54 \\
\hline $\begin{array}{l}\text { ítens lexicais não } \\
\text { utilizados no } \\
\text { tratamento }\end{array}$ & $60,2(25,9) a$ & $84,5(11,4) a$ & 0,13 & $42,2(30,2) a$ & $61,9(35,3) a$ & 0,47 & $28,0(13,3) a$ & $50,6(18,3) a$ & 0,20 \\
\hline $\begin{array}{l}\text { outras posições na } \\
\text { palavra }\end{array}$ & $56,6(38,4) \mathrm{a}$ & $32,4(38,8) a$ & 0,41 & $53,6(34,7) a$ & $51,6(39,2) a$ & 0,96 & $0,0(0,0) \mathrm{a}$ & $19,3(14,9) a$ & 0,16 \\
\hline $\begin{array}{l}\text { dentro de uma } \\
\text { classe de sons }\end{array}$ & $46,1(8,7) a$ & $20,5(18,1) b$ & 0,04 & $49,0(39,2) \mathrm{a}$ & $34,1(36,4) a$ & 0,62 & $12,1(1,4) \mathrm{a}$ & $38,3(35,0) a$ & 0,37 \\
\hline $\begin{array}{l}\text { outras classes de } \\
\text { sons }\end{array}$ & $44,3(29,9) a$ & $44,9(15,3) a$ & 0,97 & $36,3(18,5) a$ & $19,2(23,0) a$ & 0,34 & $0,0(0,0) a$ & $13,6(27,3) a$ & 0,54 \\
\hline
\end{tabular}

Legenda: PCCR: percentual de consoantes-corretas revisado; FA: fonemas adquiridos; G1: grupo 1; G2: grupo 2; A: ABAB-Retirada e Provas Múltiplas; O: Oposições Máximas Modificado; C: Ciclos Modificado.

\section{Discussão}

Detectou-se um aumento do PCC-R em todos os grupos de gravidade do desvio fonológico nos diferentes modelos pesquisados (Modelo ABABRetirada e Provas Múltiplas, Ciclos Modificado e no Modelo de Oposições Máximas), embora não tenha observado diferença estatística entre os modelos. Em um estudo ${ }^{8}$ comparando diferentes modelos de terapia demonstraram que todas as crianças aumentaram o seu percentual de consoantes corretas durante a intervenção.

Em relação às generalizações comparando os modelos nos diferentes grupos, verificou-se que os sujeitos pesquisados apresentaram evolução em relação à generalização a itens lexicais não utilizados no tratamento, para outra posição da palavra, dentro de uma classe de sons e para outras classes de sons nos modelos pesquisados (ABABRetirada e Provas Múltiplas, Ciclos Modificado e Oposições Máximas). Resultados semelhantes foram encontrados em outros estudos ${ }^{8,12-13}$.

Quando comparados os três diferentes modelos de terapia fonológica aplicados nesta pesquisa, os resultados mostraram que estes foram efetivos no tratamento das crianças estudadas, pois estas adquiriram sons em seus sistemas fonológicos e apresentaram generalizações. A eficácia desses modelos foi demonstrada em outras pesquisas ${ }^{3-4,9-}$ ${ }^{10}$. Um autor ${ }^{17}$ afirma que as abordagens fonológicas visam à generalização a partir dos alvos tratados.
Ao comparar os grupos dentro de cada modelo terapêutico, a análise estatística mostrou que o PCC$\mathrm{R}$ nos Modelos ABAB-Retirada e Provas Múltiplas e Oposições Máximas Modificado diferem estatisticamente entre o G1 e o G2. Outro estudo ${ }^{6}$ referiu que a eficácia dos tratamentos pode estar na seleção inicial do alvo de tratamento.

Quanto ao número de fonemas adquiridos no sistema fonológico, observou-se uma relação diretamente proporcional entre os grupos pesquisados, isto é, quanto maior a gravidade (G1) maiores foram as aquisições de sons, com exceção no Modelo de Ciclos Modificado. A autora de um estudo $^{5}$ enfatizou que a maior quantidade de mudança ocorreu para as crianças que tinham mais para aprender a partir da intervenção. Outro trabalho ${ }^{18}$ referiu expansão de sons excluídos no pré-tratamento no inventário de uma criança com desvio fonológico.

Em relação à generalização a itens lexicais não utilizados no tratamento, para outra posição da palavra, dentro de uma classe de sons e para outras classes de sons, quando comparadas entre os grupos, houve diferença estatística apenas para o Modelo ABAB-Retirada e Provas Múltiplas na generalização dentro de uma classe de sons. Observou-se generalização em todos os grupos com desvio fonológico nos três modelos pesquisados. Resultados semelhantes foram encontrados em vários outros estudos ${ }^{7-}$ ${ }^{8,12-13}$. Um estudo ${ }^{13}$ referiu que a generalização 
dentro de uma classe de sons e para outras classes de sons ocorreu para todos os modelos pesquisados, porém, a primeira ocorreu principalmente nos sujeitos submetidos aos Modelos de ABAB-Retirada e Provas Múltiplas e Oposições Máximas Modificado e a segunda

\section{Conclusão}

Com base nos resultados desta pesquisa verificou-se que os três diferentes modelos de terapia fonológica aplicados foram eficazes no tratamento dessas crianças. Em cada modelo houve

\section{Referências Bibliográficas}

1. Grunwell P. The nature of phonological disability in children. London: Academic Press; 1981.

2. Tyler A, Figurski R. Phonetic inventory changes after treating distinctions along an implicational hierarchy. Clin. Ling. Phon. 1994;8(2):91-107.

3. Bagetti T, Mota HB, Keske-Soares M. Modelo de oposições máximas modificado: uma proposta de tratamento para o desvio fonológico. Rev. Soc. Bras. Fonoaudiol. 2005 Jan-Mar;10(1):36-42.

4. Tyler A, Edwards ML, Saxman J. Clinical application of two phonologically based treatment procedures. J. Speech Hear. Dis. 1987;(52):393-409.

5. Williams AL. Multiple oppositions: theoretical foundations for an alternative contrastive intervention framework. Am. J. Speech-Lang. Path. 2000 Nov;9:2828.

6. Gierut JA. Complexity in phonological treatment: clinical factors. Lang, Speech Hear Serv Sch. 2001;32:229-41.

7. Mota HB, Bagetti T, Keske-Soares M, Pereira LF. A generalização em sujeitos com desvio fonológico médiomoderado tratados pelo modelo de oposições máximas. Rev. Soc. Bras. Fonoaud. 2004;9:102-11.

8. Crosbie S, Holm A, Dodd B. Intervention for children with severe speech disorder: a comparison of two approaches. Int. J. Lang. Comm. Dis. 2005 Oct-Dec;40(4):467-91.

9. Mota HB, Bagetti T, Keske-Soares M, Pereira LF. A generalização baseada nas relações implicacionais em sujeitos submetido à terapia fonológica. Pró-Fono R. Atual. Cient. 2005 Jan.-Abr;17(1):99-110. para os sujeitos submetidos ao Modelo ABABRetirada e Provas Múltiplas. Uma autora ${ }^{6}$ refere que essas duas últimas generalizações são desejáveis no tratamento por contribuírem com mudanças mais globais no sistema de sons da criança. um aumento do PCC-R, do número dos fonemas adquiridos nos sistemas fonológicos e da generalização a itens lexicais não utilizados no tratamento, outras posições na palavra, dentro de uma classe de sons e para outra classe de sons.
10. Mota HB, Keske-Soares M, Bagetti T, Ceron MI, Melo Filha MGC. Análise comparativa da eficiência de três diferentes modelos de terapia fonológica. Pró-Fono R. Atual. Cient. 2007 Jan-Abr;19(1):67-74.

11. Elbert M, Gierut JA. Handbook of clinical phonology. London: Taylor e Francis Ltda; 1986.

12. Ceron MI, Keske-Soares M. Terapia Fonológica: a generalização a itens não utilizados no tratamento (outras palavras). Rev. Cefac. 2007;9(4):453-60.

13. Ceron MI, Keske-Soares M. Terapia Fonológica: a generalização dentro de uma classe de sons e para outras classes de sons. Rev. Cefac. 2008;10(3):311-20.

14. Bernhardt B. Developmental implications of nonlinear phonological theory. Clin. Linguist. Phon. 1992;6:25981.

15. Shriberg LD, Austin D, Lewis BA, Mcsweeny JL, Wilson DL. The percentage of consonants correct (PCC) metric: extensions and reliability data. J. Speech Lang. Hear. Res. 1997;40(4):708-22.

16. Shriberg LD, Kwiatkowski J. Phonological disorders I: a diagnostic classification system. J. Speeh Hear. Dis. 1982 Aug;47:226-41.

17. Tyler A. Commentary on "Treatment decisions for children with speech-sound disorders": revisiting the past in EBP. Lang., Speech Hear. Serv. Sch.. 2006;37:280-3.

18. Barlow JA. Phonological change and the representation of consonant clusters in spanish: a case study. Clin. Linguist. Phon. 2005;19:659-79. 